Provided for non-commercial research and education use. Not for reproduction, distribution or commercial use.

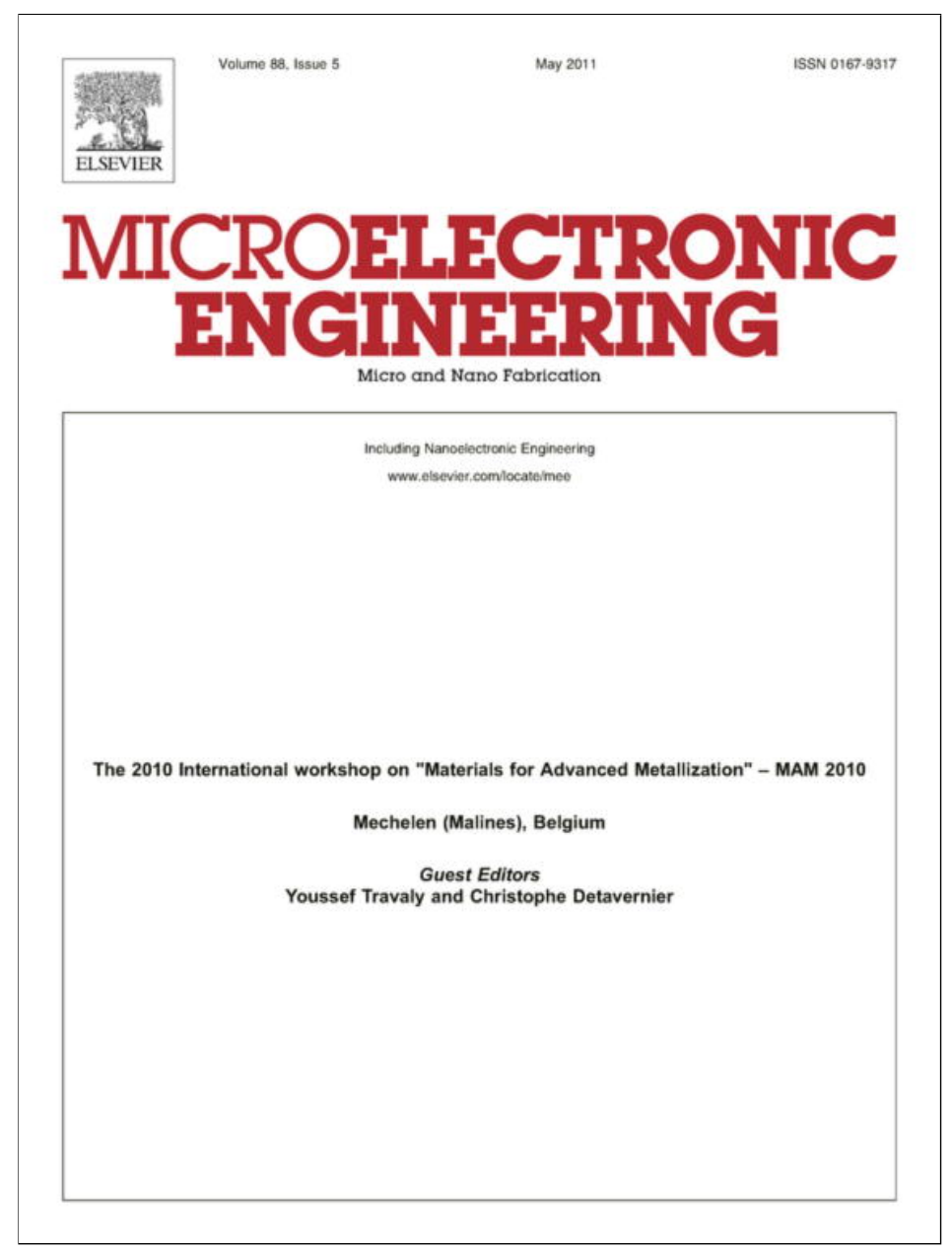

This article appeared in a journal published by Elsevier. The attached copy is furnished to the author for internal non-commercial research and education use, including for instruction at the authors institution and sharing with colleagues.

Other uses, including reproduction and distribution, or selling or licensing copies, or posting to personal, institutional or third party websites are prohibited.

In most cases authors are permitted to post their version of the article (e.g. in Word or Tex form) to their personal website or institutional repository. Authors requiring further information regarding Elsevier's archiving and manuscript policies are encouraged to visit:

http://www.elsevier.com/copyright 


\title{
Integrated diffusion-recombination model for describing the logarithmic time dependence of plasma damage in porous low- $k$ materials
}

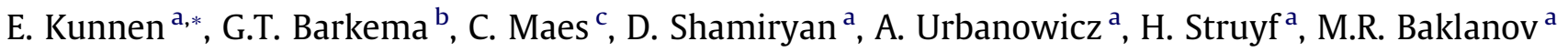 \\ a IMEC, Kapeldreef 75, 3001 Heverlee, Belgium \\ ${ }^{\mathrm{b}}$ Institute for Theoretical Physics, Utrecht University, Leuvenlaan 4, 3584 CE Utrecht, The Netherlands \\ ${ }^{\mathrm{c}}$ Instituut voor theoretische fysica, Celestijnenlaan 200D, 3001 Heverlee, Belgium
}

\section{A R T I C L E I N F O}

\section{Article history:}

Available online 11 July 2010

\section{Keywords:}

Plasma damage

Recombination

Diffusion

Low-k

Porous

\begin{abstract}
A B S T R A C T
This work proposes an extended model that describes the propagation of damage in porous low- $k$ material exposed to a plasma. Recent work has indicated that recombination and diffusion play a more dominant role than VUV light [1-5] in oxygen plasma induced damage. Especially at low depths, the radical concentration is determined by the number of radicals that disappear back into the plasma while the final depth of damage is defined by recombination of oxygen atoms. A logarithmic equation has been proposed to describe the behavior as a function of time. In this work this equation is extended to take diffusion into account, next to recombination. The results are in agreement with experimental data and one-dimensional random walk theory calculations.
\end{abstract}

(c) 2010 Elsevier B.V. All rights reserved.

\section{Introduction}

Scaling in semiconductor industry continues at the pace described in the ITRS roadmap. As a consequence, low capacitive materials, so called low- $k$ materials, have been introduced to reduce cross line talking between the interconnecting $\mathrm{Cu}$ lines. During integration, these materials are exposed to plasmas. Such exposure can lead to a degradation of the low capacitive property, which is commonly denoted by the term 'plasma damage'. Plasma damage is characterized by the removal of hydrophobic carbonhydrogen groups and absorption of water, resulting in a high $k$ value. Next to VUV light, the diffusion and recombination of radicals entering the porous low- $k$ material are assumed to be the main causes for plasma damage. Recent research has shown that the importance of diffusion and recombination by oxygen atoms in porous SiOC materials [2] can contribute more to plasma damage than the VUV interaction. In certain oxygen plasma-damaged porous $\mathrm{SiOCH}$ samples [1], the observed depth of damage has been explained to be recombination-limited and to be corresponding to a logarithmic equation $[1,6]$ :

$$
x(t)=A_{1} * \ln \left(t / A_{2}+1\right)
$$

The empirically observed Eq. (1) describes the position $x(t)$ of a steep damaged/undamaged interface that penetrates into the sample with time $t$. The speed of penetration is proportional to the

\footnotetext{
* Corresponding author. Tel.: +32 16 288504; fax: +32 16281214 .

E-mail address: eddy.kunnen@imec.be (E. Kunnen).
}

amount of radicals at this interface. $A_{1}$ is the distance over which the concentration of radicals is dropped by a factor 1/e through recombination inside the damaged layer. The recombination goes proportional with the local radical concentration following a Langmuir-Hinshelwood based surface mediated recombination scheme. Secondly, in time frame $A_{2}$ the concentration of radicals at the penetrating damaged/undamaged interface is dropped by a factor of 2 .

Nevertheless diffusion is expected to also play a role [4]. Therefore in the first part of this paper, certain aspects of diffusion will be addressed by random walk calculations while in the second part a model is set up taking into account recombination and diffusion. Finally, in the third part we will show that the model agrees with the random walk calculations and experimental data from literature.

\section{Random walk calculations}

Eq. (1) has been experimentally observed and explained in Refs. $[1,6]$ and implies that the oxygen radical concentration drops exponentially inside the damaged material. It describes the experimental data more accurately than a diffusion based Deal Grove type model. The exponential drop of the oxygen radical concentration is explained as radicals recombining into oxygen molecules through Langmuir-Hinshelwood surface reactions inside the damaged layer. Finally, the exponential drop of the radical concentration is confirmed by random walk calculations. The onedimensional random walk model consists of setting the interface 


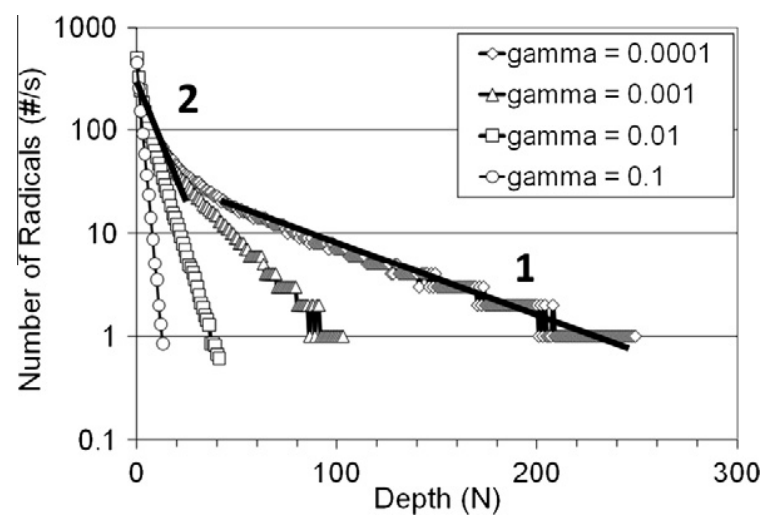

Fig. 1. The amount of radicals reaching the pristine interface at position $N$ as function of different recombination probabilities (gamma). If the pristine interface $(N)$ is close to the plasma surface (at $N=0$ ), a steep drop is observed (2) before a constant exponential drop is observed (1).

with the pristine interface at depth $N$ and simulating a random walk of oxygen radicals from the surface of the damaged layer to the interface with the pristine layer. The radicals have an equal probability of walking in both directions along a one-dimensional axis: they can walk back into the plasma and disappear, or arrive at the pristine material and react or recombine into an oxygen molecule with a recombination probability $\gamma$. At small $N$, the radical concentration drops faster before arriving in the exponential range, see Fig. 1. The reason is explained in Fig. 2.

Fig. 2 shows the mechanism by which the radicals are consumed for an arbitrary recombination value of 0.0001 . For any position of the pristine interface $N$, most of the radicals walk back into the plasma. However, from a certain depth onwards this value remains constant and recombination determines the number of radicals that arrive at the pristine material and react. From this point of view, Eq. (1) which is solely based on recombination should only be applied for larger $N$ where recombination is dominant. For small $N$ and relatively short times, a diffusion type of mechanism, the walk-back into the plasma dominates the number of radicals that reach the pristine material. Nevertheless Eq. (1) accurately fits the data in experiments [6] indicating that only large times are probed during experiments. The random walk calculations are done assuming sufficiently fast diffusion so a radical ends up either at the interface, or walks back into the plasma or recombines. Even in these conditions the calculations show that for short times a diffusive component needs to be taken into account that counts for the walk back into plasma. Finally, setting

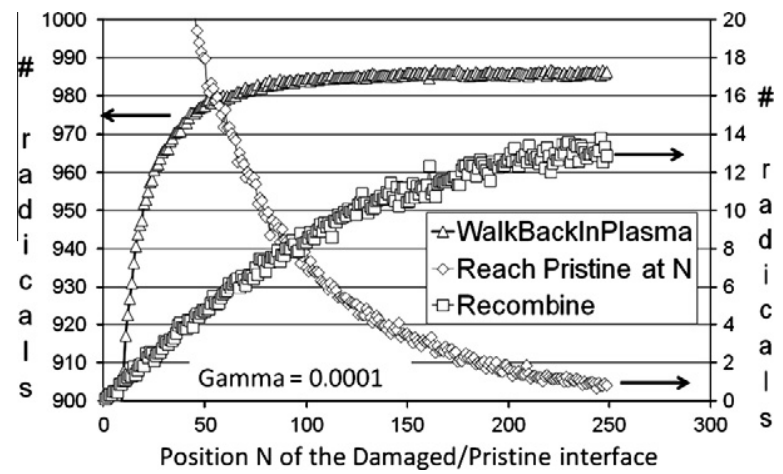

Fig. 2. Most of the radicals walk back into the plasma. From a depth of about 50, the amount that walks back into the plasma remains constant and the amount of arriving radicals that arrive at the pristine interface $(N)$ is determined by recombination.

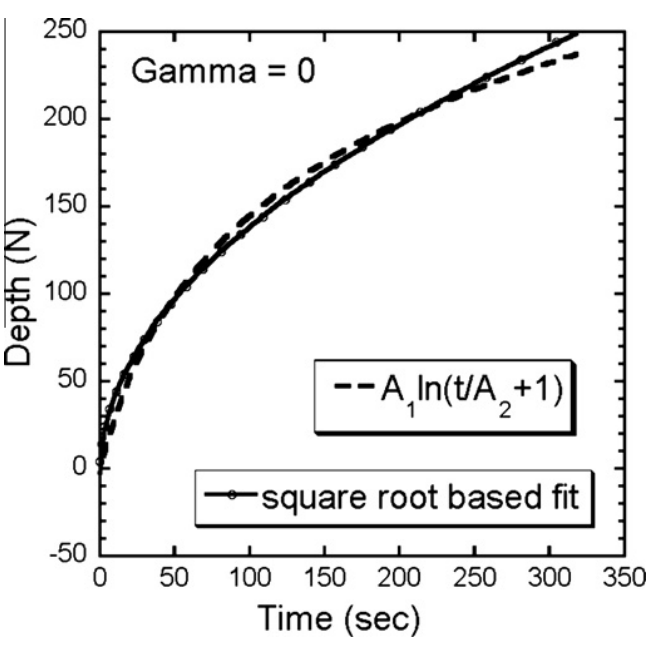

Fig. 3. When the recombination probability in the random walk calculations is set to zero, Eq. (1) fails and a square root dependency of the time appears.

$\gamma=0$ in the random walk calculations allows to calculate the oxygen radical concentration profile solely determined by walk back into plasma and reaction with the pristine material. From this concentration profile a qualitative damage depth-time relationship can be established, Fig. 3, taking, e.g., a consumption of 10 radicals/step for converting pristine into damaged material. A common condition of 1000 radicals arriving/second at the surface is used as a starting-point.

If $\gamma=0$, the position of the interface with the pristine material cannot be described accurately by (1) but can be fitted easily using the square root of time which is typical for diffusion. In summary, even in the case that diffusion speed is not the time-limiting step it needs to be taken into account because of the radicals that walk back into the plasma, especially for small $N$ and relatively short times.

\section{An integrated diffusion-recombination model}

At low pressure ( $\mathrm{mT}$ range) the mean free path of molecules is of the order of $\mathrm{mm}$ and therefore there is no chance of any gas phase interaction between radicals in the pores. The radicals perform a random walk independent of each other. Nevertheless, transport of particles is concentration-dependent under these circumstances. Moreover, exactly because of the non-interacting character there will be more particles walking from a high concentration area to an area of lower concentration than in the other direction. This type of Knudsen diffusion in porous media is described by Mason and Malinauskas [7]. They found that the flow is proportional to the concentration gradient similar to Fick's first diffusion law. However, the underlying physics is clearly different. The model proposed here incorporates Knudsen diffusion of free oxygen atoms into the porous material. Secondly, the oxygen atoms can react with the carbon-hydrogen groups and remove them. Thirdly, the oxygen atoms can recombine into oxygen molecules through Langmuir-Hinshelwood or Eley-Rideal surface reactions.

For describing the process, three densities are considered, $\rho_{f}(x$, $t), \rho_{a}(x, t), \rho_{c}(x, t)$, which are resp. free oxygen atoms, chemi-adsorbed oxygen atoms, and carbon concentration. The Knudsen diffusion of the free oxygen radicals into the porous material is described by a diffusion constant $D$. Free oxygen atoms turn into chemi-adsorbed oxygen radicals at a rate $r_{a}$. Free oxygen radicals react with bonded oxygen radicals to form $\mathrm{O}_{2}$ at a rate $r_{f a}$. Thirdly, free oxygen radicals react with carbon at a rate $r_{f c} . \rho_{f}(x, t=0)=\delta_{x, 0}$, 
$\rho_{a}(x, t=0)=0, \rho_{c}(x, t=0)=1$ are used as initial conditions at time zero while $\rho_{f}(x=0, t)=1$ is kept at all times. Following the above reaction schemes, the differential equations are:

$\underset{f}{\dot{\rho}}=D \frac{\partial^{2}}{\partial x} \rho_{f}-r_{f a} \rho_{f} \rho_{a}-r_{f c} \rho_{c} \rho_{f}-r_{a} \rho_{f}$

$\dot{\rho}_{c}=-r_{f c} \rho_{c} \rho_{f}$

$\dot{\rho}=r_{a} \rho_{f}-r_{f a} \rho_{f} \rho_{a}$

The first term in Eq. (2) describes the Knudsen diffusion, the subsequent three terms correspond to resp. the loss of free oxygen radicals by recombination, reaction with carbon, and adsorption on the surface. Secondly, the carbon concentration (3) can only drop because of reaction with free oxygen radicals. Thirdly, the amount of adsorbed oxygen radicals (4) is determined by the adsorption and the recombination. Simulation of the differential equations was done for arbitrary values $r_{f c}=1, r_{a}=0.1, r_{f a}=1, D=1.0$ for times 10-2000. Most interesting is that the carbon concentration $\rho_{c}$ collapses as a function of the variable $x / \ln (t+2 * \operatorname{sqrt}(t))$ (see Fig. 4 ) i.e., this relation is found to identify the damaged layer $l(t)$ with $x(t)$ in such a way that $\rho_{c}(x, t)=0.5$.

Therefore, taking diffusion into account, Eq. (1) could be extended to:

$x(t)=A_{1} \ln \left(t / A_{2}+\sqrt{t} / A_{3}+1\right)$

Intuitively, Eq. (5) can be explained in the following way. The speed at which the interface propagates is proportional to the radical concentration at the interface. Given that the radicals diffuse independently from each other, the radical concentration at the interface at position $x(t)$ can be written from the viewpoint of one radical. Actually the chance that the radical arrives at position $x(t)$ is, because of the recombination, related to the time $t^{*}$ the radical needs to get to position $x(t)$. Thus:

$\frac{d x}{d t} \sim \exp \left(-t^{*}\right)$

Still $t^{*}$ needs to be expressed as function of $x(t)$ in order to solve (6). A first approximation is the ballistic approximation, $t^{*} \sim x(t)$, which says there is an effective velocity $v_{\text {eff. }}$ Secondly, in order to take into account the diffusion the relationship becomes:

$x(t)=v_{\text {eff }} t^{*} \pm \sqrt{D t^{*}}$

Eq. (7) is of second order in $t^{*}$ and the positive solution will result in the relationship (5). For the exact relationship between the recombination probability and the diffusion constant on the one hand, and the fitting coefficients $A_{1}, A_{2}, A_{3}$ on the other hand, more

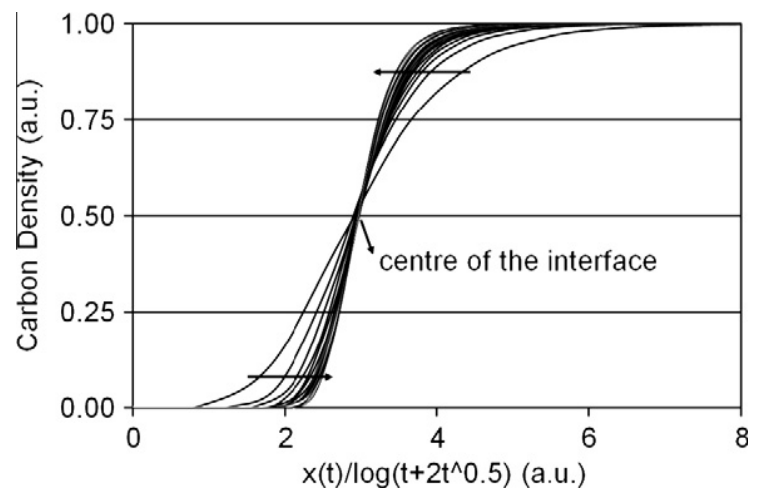

Fig. 4. The centre of the damaged/pristine interface follows $\ln (t+2 * \operatorname{sqr}(t))$.

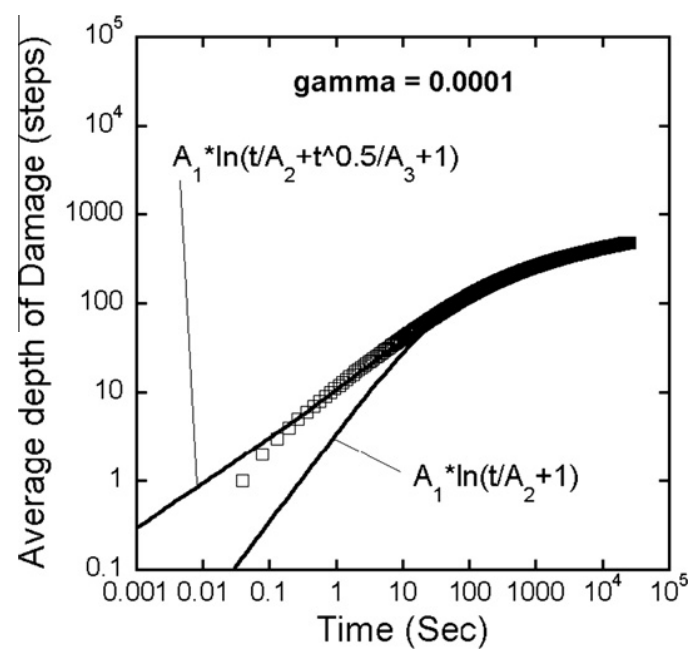

Fig. 5. A time-damage depth relationship has been generated by random walk calculations. Eq. (1), which is solely based on recombination, fails for short times while Eq. (5) allows to describe the behavior from the first points onwards.

calculations need to be performed. This is subject of possible future work. In Eq. (1) $A_{1}$ is the depth over which the radical concentration drops by a factor $1 / e, A_{2}$ is the time it takes before the radical concentration at the interface is halved. In Eq. (5) similar relationships are expected and $A_{3}$ should be related to diffusion.

\section{Verification of the model with random walk calculations and experimental data}

In the chapter on random walk calculations it has been shown that for short times the radical concentration is mainly determined by radicals walking back into the plasma. Eq. (1) is expected to fail in this regime since it is based upon recombination. Setting the recombination probability $\gamma$ to 0.0001 and taking an arbitrary value of 10 radicals needed per step to convert the pristine material into a damaged layer, a relationship between time and damage depth can be established. The interface is sharply defined at position $N$. A common condition of 1000 radicals arriving at the surface every second is assumed. Fig. 5 shows the depth of damage as a function of time under these simulation conditions. Clearly, Eq. (1) does not fit the range below $10 \mathrm{~s}$ while above $10 \mathrm{~s}$ a good

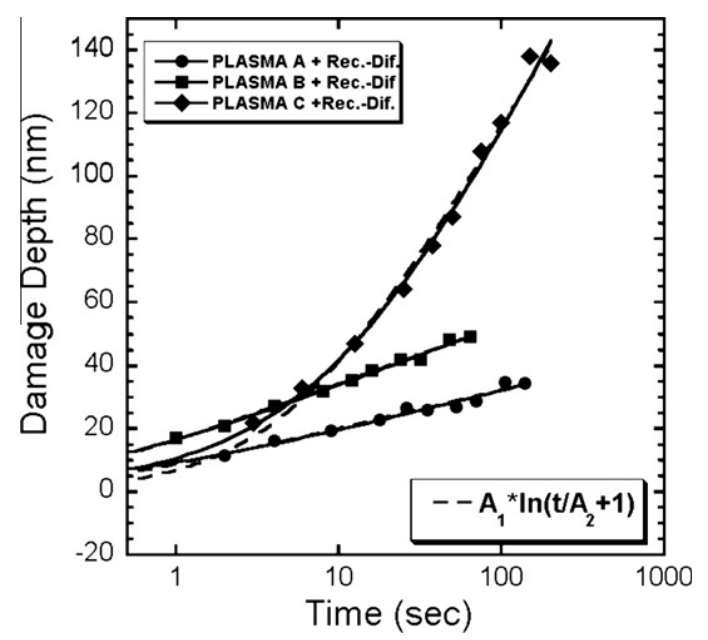

Fig. 6. Experimental data taken from literature and fitted by the two equations. Only for plasma condition C the fit is more accurate for Eq. (5) than Eq. (1). 
Table 1

Values obtained for $A_{12,3}$ by fitting the experimental data of Fig. 6 using Eqs. (1) and (5). $A_{2}$ and $A_{3}$ are of the same order indicating the importance of diffusion for short times.

\begin{tabular}{lllllll}
\hline & \multicolumn{2}{l}{$A_{1} * \ln \left(\mathrm{t} / A_{2}+1\right)$} & & \multicolumn{3}{l}{$A_{1} * \ln \left(\mathrm{t} / A_{2}+t^{0.5} / A_{3}+1\right)$} \\
\cline { 2 - 3 } \cline { 5 - 6 } & $A_{1}(\mathrm{~nm})$ & $A_{2}(\mathrm{~s})$ & & $A_{1}(\mathrm{~nm})$ & $A_{2}(\mathrm{~s})$ & $A_{3}\left(\mathrm{~s}^{0.5}\right)$ \\
\hline Plasma A & 5.4 & 0.24 & & 6 & 0.4 & 0.6 \\
Plasma B & 8.2 & 0.15 & & 9 & 0.2 & 0.8 \\
Plasma C & 38 & 5.1 & & 46 & 10 & 6 \\
\hline
\end{tabular}

correlation is found. Eq. (5) however, describes the graph down till the first initial points.

Finally, the question remains to which extent the generated model fits the experimental data, Fig. 6, taken from literature [3] (replot of graph 7 in Ref. [3]). Eq. (1) has been shown to adequately describe damage as a function of time $[1,6]$. The data are repeated in Fig. 6 to show the improvement gained by Eq. (5). Only for plasma condition $\mathrm{C}$, an improvement is found at short times. The corresponding values of the fitting parameters are listed in Table 1. The large difference in values for plasma A, B compared to $C$ is related to the higher recombination coefficient resulting from plasma $A$ and $B$ because of ion bombardment [3]. Similar values are obtained for Eqs. (1) and (5) for $A_{1}$ and $A_{2} . A_{3}$ is of the same order, indicating that diffusion plays a role for small times.

\section{Conclusion}

In this paper the role of recombination and diffusion of radicals inside a plasma-damaged porous SiOCH low- $k$ layer is investigated.
It is concluded that the effect of oxygen radicals walking back into the plasma may impact the way the damage progresses into the material. Especially for short times, diffusion plays a role while for longer exposure times the depth of damage is limited by the recombination of radicals inside the damaged layer. A new model that incorporates Knudsen diffusion is proposed and discussed. Finally, an equation that describes the time dependency of damage propagating into the material, taken diffusion and recombination into account is proposed and evaluated by experimental data and random walk calculations. The exact relationship between the fitting coefficients and diffusion and recombination needs further research.

\section{References}

[1] E. Kunnen, A. Urbanowicz, D. Shamiryan, H. Bender, A. Franquet, H. Struyf, W. Boullart, M.R. Baklanov, in: 56th American Vacuum Society Conference, November 8-13, 2009, San Jose, California, p. 78 (Abstract Book PS-TuA7).

[2] E. Hudson, M. Moravej, M. Block, S. Sirard, D. Wei, K. Takeshita, in: AVS 2009, San Jose California, 56th American Vacuum Society Conference, November 813, 2009, San Jose, California, p. 190 (Abstract Book PS2-ThM11).

[3] E. Kunnen, M.R. Baklanov, A. Franquet, D. Shamiryan, T.V. Rakhimova, A.M Urbanowicz, H. Struyf, W. Boullart, J. Vac. Sci. Technol. B 28 (3) (2010) 450-459.

[4] M.A. Goldman, D.B. Graves, G.A. Antonelli, S.P. Behera, J.A. Kelber, J. Appl. Phys. 106 (2009) 013311.

[5] J. Kelber, S. Behera, S. Manandhar, S. Gaddam, in: San Jose California, 56th American Vacuum Society Conference, November 8-13, 2009, San Jose California, p. 9 (Abstract Book PS1-MoM3).

[6] E. Kunnen, to be published.

[7] E.A. Mason, A.P. Malinauskas, Gas Transport in Porous Media - The DustyGasModel, Elsevier, Amsterdam, 1983. 